\title{
Coadsorption of Low-Molecular Weight Aromatic and Aliphatic Alcohols and Acids with the Cationic Surfactant, CTAB, on Silica Surfaces
}

\author{
Thipvaree Wangchareansak, ${ }^{\dagger,}$ Max A. Keniry, ${ }^{\S}$ Guangming Liu, " and Vincent S. J. Craig*,†,॥ \\ ${ }^{\dagger}$ Department of Applied Mathematics, Research School of Physics and Engineering, Australian National University, Canberra 0200, \\ ACT, Australia \\ ${ }^{\ddagger}$ Department of Chemistry, Faculty of Science, Kasetsart University, Bangkok, Thailand 10900 \\ ${ }^{\S}$ Research School of Chemistry, Australian National University, Canberra 0200, ACT, Australia \\ "Department of Chemical Physics, Hefei National Laboratory for Physical Sciences at the Microscale, University of Science and \\ Technology of China, Hefei 230026, P. R. China
}

Supporting Information

ABSTRACT: We have investigated the coadsorption of a range of small molecules with the cationic surfactant $\mathrm{CTAB}$ to silica surfaces over a range of concentrations and $\mathrm{CTAB}$ to solute ratios and compared the coadsorption with adsorption in the presence of the salicylate ion. We find that molecules with aromatic character and molecules with double bonds are most favorably adsorbed, and we attribute this to cation $-\pi$ bonding between the surfactant headgroups and the $\pi$ orbitals of the unsaturated bonds of the solute molecules. The adsorption is complex and depends on chemical interactions between the solute molecules and the surfactant, which are highly specific to the structure of the solute. To improve our understanding of the specifics of

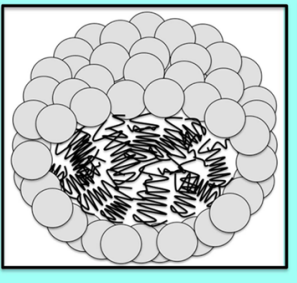

CTAB

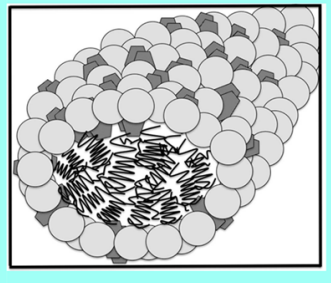

$\mathrm{CTAB}+$ Salicylate these interactions, we have performed one-dimensional rotating frame Overhauser spectroscopy (ROESY) nuclear magnetic resonance experiments. These experiments show the complexity of the intermolecular interactions and can be used to determine the position of the solute molecule with regard to the CTAB molecules in the adsorbed aggregates. The ROESY spectrum for the salicylate anion is distinct from those of the other solute molecules and suggests that the anions are dimerizing. Along with the cation $-\pi$ bonding between the dimers, this provides a model for the strong influence that salicylate has on adsorption, micellar structure, and viscoelasticity. The ROESY data indicate that the catechol molecule interacts with all parts of the surfactant alkane chains such that they wrap around the molecule, but this has little effect on the interfacial curvature or aggregate shape. More intense isophthalic acid-CTAB intermolecular ROEs compared to those of other aromatic solutes are consistent with an interaction between isophthalic acid and the headgroups of two surfactant molecules that slows the intramicellar motion of isophthalic acid. Differences in interactions between solute molecules and the aliphatic surfactant chains do not result in changes in micelle structure.

\section{INTRODUCTION}

The adsorption of surfactant on solid surfaces plays a critical role in detergency, froth flotation, wetting, and surface passivation. Hence, the process of adsorption and the morphology of adsorbed surfactants have been studied extensively. In many applications, surfactants are paired with other small molecules to modify the properties of the solution. In this case, the hydrophobic cores of the admicelles generally play a significant role in the coadsorption or adsolubilization of other molecules and will lead to significant adsorption of hydrophobic molecules onto hydrophilic surfaces that would not otherwise take place. ${ }^{1}$

The salicylate ion is commonly used with tetramethyl alkylammonium surfactants in formulations as it has a strong influence on the aggregate structure, promoting a transition to wormlike micelles at low surfactant concentrations, which results in dramatic changes in rheological properties, in particular an increased viscosity. Despite the commercial importance of these mixtures, the interaction of salicylate with $\mathrm{CTAB}$ micelles is not completely understood, and subtle alterations in the molecular architecture have substantial effects. For example, sodium salicylate, salicylic acid (2-hydroxybenzoic acid), 3-chlorobenzoate, and 4-chlorobenzoate are effective at promoting the formation of wormlike micelles and inducing viscoelasticity, whereas 2-chlorobenzoate, 3-hydroxybenzoic acid, and 4-hydroxybenzoic acid are not, even though the molecules differ only in the position of the hydroxyl group. ${ }^{2-4}$ Even though the position and orientation of solute molecules have been studied by several techniques such as nuclear magnetic resonance (NMR), ${ }^{5,6}$ voltammetry, ${ }^{7}$ theoretical, ${ }^{8}$ and

Received: March 31, 2014

Revised: $\quad$ May 20, 2014

Published: May 21, 2014 
simulation studies, ${ }^{9}$ there is no general agreement about the position, orientation, or interaction between $C T A B$ and the salicylate anion that so strongly influences the micelle structure and thereby the properties of the fluid.

Here we explore the adsorption of mixtures of $\mathrm{CTAB}$ and a range of low-molecular weight organic acids and alcohols to determine if the solutes have a strong influence on the amount of adsorption and whether this can be understood in terms of the molecular architecture of the solute. These molecules have hydrophobic regions that are both aromatic and nonaromatic and have hydrophilic groups that differ in their type, number, and location. We have also studied the position, orientation, and interaction of the solute molecules in surfactant micelles by two-dimensional (2D) NMR.

It is known that different surfaces influence, or template, surfactant adsorption to differing degrees. ${ }^{10-12}$ Hydrophobic surfaces are readily covered at low surfactant concentrations by a monolayer of surfactant. When the hydrophobic substrate exhibits strong ordering, the film adopts a structure that is strongly templated by the substrate, such as when highly ordered pyrolitic graphite (HOPG) is employed as the substrate. ${ }^{13}$ Hydrophilic surfaces generally result in adsorbed surfactant structures that are bilayered (admicelles) to minimize exposure of hydrophobic surfactant chains to water. At concentrations above the critical micelle concentration $(\mathrm{cmc})$, these admicelles to some degree reflect the micelle structures present in solution at higher concentrations, depending on the strength of the templating action of the substrate. Mica, which is crystalline and atomically flat, is known to strongly influence the ordering of adsorbed cationic surfactant films. ${ }^{13,14}$ Silica has a comparatively weak templating effect and therefore less influence on the structure of the adsorbed films. ${ }^{10,15}$ As such, the adsorbed structures on silica more closely reflect the aggregate structures present in solution at slightly higher concentrations. The solutes that are added to the solution influence the structure of micelles and are also likely to influence admicelle structure and therefore alter the adsorption of the surfactant at surfaces. Indeed, we have seen evidence of this with salicylate. ${ }^{12}$ We have chosen silica as a substrate, as it is a weakly templating surface and therefore will not dominate the structural form that the admicelles adopt.

\section{EXPERIMENTAL SECTION}

Materials. All water used in the experiments was filtered through a Millipore Gradient Filtration system. CTAB was obtained from SigmaAldrich with a purity of $>99 \%$ and further purified by recrystallization twice from an acetone/methanol mixture. Sodium salicylate, benzoic acid, catechol, citric acid, fumaric acid, isophthalic acid, maleic acid, trans-trans muconic acid, phenol, and phthalic acid of AR grade $(\geq 99.9 \%)$ were purchased from Sigma-Aldrich, Fluka, and Merck. Silicon wafers with a surface roughness of $0.6 \mathrm{~nm}$ RMS $(10 \mu \mathrm{m} \times 10$ $\mu \mathrm{m}$ ) and a customized $319 \mathrm{~nm}$ thick thermally grown oxide layer were used in the adsorption studies (Silicon Valley Microelectronics, Santa Clara, CA). The wafers were diced into $50 \mathrm{~mm} \times 10 \mathrm{~mm}$ pieces and cleaned using purified ethanol and an aqueous $10 \%(\mathrm{w} / \mathrm{w}) \mathrm{NaOH}$ solution before being rinsed thoroughly with water before use. Buffers were not used to control the $\mathrm{pH}$ of the solution as ions introduced with the buffers can bind with the surfactant headgroups and strongly influence the self-assembly properties, whereas the $\mathrm{pH}$ change induced by the acids themselves will have a very minor effect on surfactant selfassembly. Therefore, when the additive is an acid, the $\mathrm{pH}$ of the solution will depend on the concentration of the acid and can be calculated from tabulated $\mathrm{p} K_{\mathrm{a}}$ values.

Optical Reflectometry (OR). The use of optical reflectometry (OR) to study adsorption was proposed by Dijt et al. ${ }^{16}$ in 1994 . The
OR technique is a form of ellipsometry in that it relies on the change in the reflected polarization of light from a substrate that takes place upon adsorption and is suitable for the study of small molecules, surfactants, polymers, and nanoparticles $(<100 \mathrm{~nm})$. The details of a purpose built OR used for our measurements have been described previously ${ }^{10,12}$ and demonstrated to have excellent resolution and stability ${ }^{17,18}$ Briefly, linear polarized light from a He/Ne laser enters the liquid cell through a $45^{\circ}$ glass prism that contains the solution of interest and impinges on the surface to be studied. The reflected light leaves the cell before being split into two components, being the parallel ( $\mathrm{p}$ ) and perpendicular (s) polarizations. Both components are converted into a voltage using matched detectors and recorded using LabView (National Instruments). To obtain the surface excess $(\Gamma)$, the optical signal is converted from the measured $\mathrm{p}$ and $\mathrm{s}$ intensities to the surface excess $(\Gamma)$ according to

$$
\Gamma=\Delta S / S_{0} A_{\mathrm{S}}
$$

where $\Gamma$ is the adsorbed amount, $S_{0}$ is a ratio of reflectivity of the two components of the reflected light $(\mathrm{p} / \mathrm{s})$ at time zero, and $\Delta S$ refers to the change in $S$ with time $\left(S-S_{0}\right)$. The sensitivity parameter, $A_{S}$, is calculated from a multilayer Fresnel optical model using the matrix method of Abelès ${ }^{19}$ by taking the refractive indices of water, silica, and silicon to be $1.33,1.46$, and 3.8 , respectively, and using a $\mathrm{d} n / \mathrm{d} c$ value for CTAB and the solute molecules. These are listed in Table $S 1$ of the Supporting Information. A silicon wafer with a $320 \mathrm{~nm}$ oxide layer was used as the substrate to provide a high level of sensitivity and insensitivity to the alignment error. All $\mathrm{d} n / \mathrm{d} c$ values were determined by measuring the slope of the refractive index versus concentration for a series of solutions at different concentrations using an Optilab T-rEX refractometer. It is important to note that the OR is arranged such that the data provide a measure of the mass areal density (milligrams per square meter) of the adsorbed material and are insensitive to the configuration of the material at the surface. Material is delivered to the surface via a port in the prism, resulting in a stagnant point at which the surface is interrogated. At the stagnant point, there is no flow and material is transported to the surface by diffusion.

All OR experiments were performed in a temperature-controlled room at $25 \pm 0.1{ }^{\circ} \mathrm{C}$. When working with $\mathrm{CTAB}$, one must ensure that the temperature of the experiment be maintained above the Krafft temperature. For all the experiments, the solutions used, including the concentrated stock solutions used to prepare them, were stored in the same room as the instrument for several days to ensure thermal equilibration. In this way, we could also ensure that the temperature of the experiment always exceeded the Krafft temperature. If stored below the Krafft temperature, the stock solution would show clear evidence of precipitation of $\mathrm{CTAB}$. All measurements were repeated at least three times and conducted without $\mathrm{pH}$ adjustment.

Nuclear Magnetic Resonance (NMR). Surfactant solutions for NMR experiments were prepared using $\mathrm{D}_{2} \mathrm{O}$ with $2 \mathrm{mM} \mathrm{CTAB}$ and 2 $\mathrm{mM}$ solute. All NMR spectra were recorded at $25{ }^{\circ} \mathrm{C}$ on an Agilent (formerly Varian) INOVA 500 spectrometer operating at a ${ }^{1} \mathrm{H}$ frequency of $500.0 \mathrm{MHz}$ and equipped with a $z$-axis pulsed field gradient indirect detection probe. The one-dimensional (1D) DPFGSE-ROESY (double pulsed field gradient spin echo-rotating frame nuclear Overhauser effect spectroscopy) and 2D zTOCSY (ztotal correlation spectroscopy) experiments were conducted using Agilent ChemPack pulse sequences. Selective excitation in the 1D ROESY experiment was achieved with two Q3-shaped pulses within the DPFGSE element. Each 1D ROESY spectrum was acquired with 4096 complex points over a sweep width of $4800 \mathrm{~Hz}$ with a $1.5 \mathrm{~s}$ recycle time and a $200 \mathrm{~ms}$ mixing period. The transverse ROESY (TROESY $)^{20}$ spin lock was $\gamma B_{1} / 2 \pi=4.9 \mathrm{kHz}$. The spectra were zero filled to 8192 complex points prior to exponential function weighting and Fourier transformation. Peak areas were quantitated using the deconvolution routine in iNMR Reader (http://www.inmr.net) and normalized by division of the autopeak. A typical 2D TOCSY (total correlation spectroscopy) experiment was conducted with 2048 complex $t_{2}$ points and $256 t_{1}$ increments over a sweep width of $4800 \mathrm{~Hz}$ in both dimensions. The recycle time was $1 \mathrm{~s}$, and the DIPSI2 mixing period was $30 \mathrm{~ms}$. The resolution in $f_{1}$ was improved by 
doubling the number of points by linear prediction followed by zero filling to 1024 real points prior to Gaussian weighting and 2D Fourier transformation.

\section{RESULTS AND DISCUSSION}

OR Experiments. A typical OR experiment is begun by recording the baseline in the presence of the solvent without surfactant or solute, and this baseline is used to define the level of zero adsorption. In this experiment, we used pure water to obtain baseline measurements. When the solution with surfactant is added, the signal shows an increase in surface excess, which saturates at a stable value. After the signal reaches a constant value, the surfactant can be washed from solution by the reintroduction of water. The surface excess is observed to decrease, and in a system without an adsorbing contaminant and temperature drift, the signal returns to the baseline value as shown in the inset of Figure 1. The inset shows the adsorption of $1 \mathrm{mM} \mathrm{CTAB}$ in water to silica as a function of time. The equilibrium surface excess obtained is $2.0 \pm 0.1 \mathrm{mg} / \mathrm{m}^{2}$.

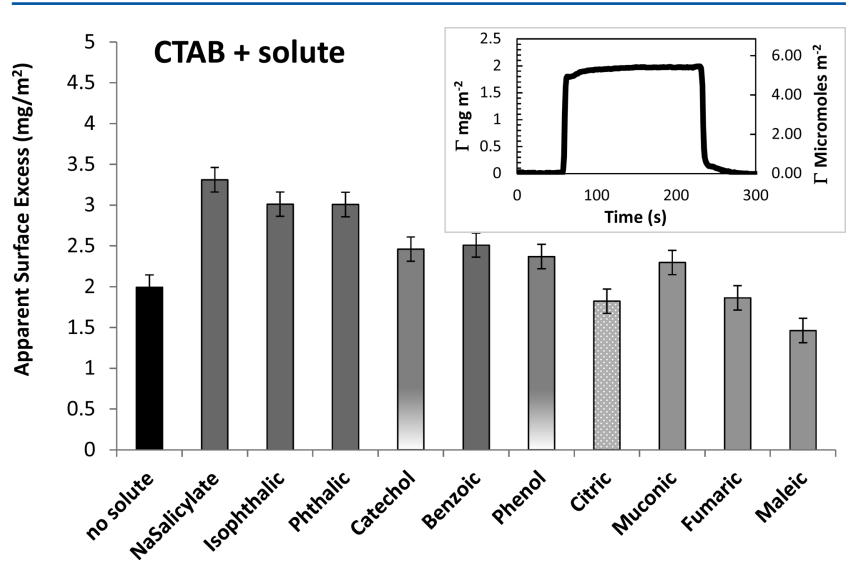

Figure 1. Apparent surface excess due to adsorption from a solution of $1 \mathrm{mM}$ CTAB with $1 \mathrm{mM}$ solute. The apparent adsorption was calculated using the sensitivity parameter for $\mathrm{CTAB}\left(A_{\mathrm{S}}=-0.02195\right)$. Data for aromatic solute molecules are colored darker gray. Data for solutes without acid groups are shown with a gradient in color, and data for the citric acid solute are shown with a pattern to indicate it lacks any $\mathrm{C}=\mathrm{C}$ groups. The error bars reflect the uncertainty in the absolute surface excess due to instrument alignment. This is larger than the repeatability of the measurements. The inset shows optical reflectometry $(\mathrm{OR})$ data for the adsorption of $\mathrm{CTAB}$ to silica in real time. A stable baseline was obtained before introduction of the surfactant solution ( $1 \mathrm{mM} \mathrm{CTAB})$ into the cell at $\sim 60 \mathrm{~s}$. At approximately $220 \mathrm{~s}$, the surfactant solution was removed by rinsing the sample with the pure solvent, in this case pure water. The signal then returns to the baseline. The equilibrium surface excess measured is $2.0 \pm 0.1 \mathrm{mg} / \mathrm{m}^{2}$.

By performing similar experiments at a number of concentrations, we can construct an adsorption isotherm. At $1 \mathrm{mM}$ CTAB, we are above the critical micelle concentration (cmc) and therefore also above the concentration at which the maximal surface coverage is obtained, which is the maximal coverage concentration $(\mathrm{mcc})$. Any further increase in $\mathrm{CTAB}$ concentration will not result in any increase in surface concentration. The adsorption isotherm for CTAB obtained using OR can be found in our previous work. ${ }^{21}$ Similar experiments can be conducted in which instead of $\mathrm{CTAB}$ alone being introduced, $\mathrm{CTAB}$ and an another molecule (or solute) are introduced into the fluid cell. The addition of a solute will in general have no influence or reduce the $\mathrm{cmc}$ and the mcc, and moreover, different solutes can have very different effects on the cmc. By working above the $\mathrm{cmc}$, we can avoid the complications caused by shifts to lower the $\mathrm{cmc}$ and focus on changes in the maximal surface excess as the solutions remain above the $\mathrm{cmc}$ at all times. The adsorption of each of the solutes in the absence of $\mathrm{CTAB}$ was measured by $\mathrm{OR}$ and found to be $<0.05 \mathrm{mg} / \mathrm{m}^{2}$ in every case (Table $\mathrm{S} 3$ of the Supporting Information).

The effect of solute on the apparent surface excess of the $\mathrm{CTAB} /$ solute mixture is shown in Figure 1. The solutes in this experiment have a range of chemical motifs such as aromaticity, carboxyl groups, hydroxyl groups, and $\pi-\pi$ conjugation. In these experiments, the change in the polarization of reflected light is converted to surface excess using the sensitivity parameter calculated for CTAB, and then we obtain what we call the apparent surface excess as shown in Figure 1. We call this the apparent surface excess because the change in signal is likely due to adsorption of both CTAB and the solute. As the solute has a different $\mathrm{d} n / \mathrm{d} c$, this will introduce an error. Additionally, we note that from these data it is not possible to discern how much of the adsorption can be attributed to CTAB and how much can be attributed to the solute. What is apparent is that the data in Figure 1 show that in comparison to CTAB alone the surface excess is increased by most solutes but not all solutes. The solutes that lead to an increase in surface excess are $\mathrm{NaSal}$, phthalic acid, isophthalic acid, benzoic acid, catechol, phenol, and muconic acid. Most of those molecules are benzene derivatives with the exception of muconic acid. While the combined surface excess of $\mathrm{CTAB}$ and solute generally exceeds that of $\mathrm{CTAB}$ alone, below we show that coadsorption actually reduces the amount of $C T A B$ on the surface and the general increase in surface excess is due to the amount of solute overcompensating for this loss. Overcompensation is not always the case as seen for citric acid and maleic acid.

The acids, citric, muconic, fumaric, and maleic, have a weaker influence on the apparent surface excess. Citric acid lacks any double bonds and therefore cannot participate in $\pi$-cation bonding with the tetramethylammonium headgroup; therefore, one might expect a reduced level of adsorption. In comparing fumaric, maleic, and muconic acids, we note that muconic acid has an additional methylene group and as such has more hydrophobic character, which should lead to greater coadsorption. The differences between the trans and cis isomers, fumaric and maleic acid, are likely related to differences in $\mathrm{p} K_{\mathrm{a}}$ (for fumaric acid, $\mathrm{p} K_{\mathrm{a} 1}=3.03$ and $\mathrm{p} K_{\mathrm{a} 2}=$ 4.44; for maleic acid, $\mathrm{p} K_{\mathrm{a} 1}=1.9$ and $\left.\mathrm{p} K_{\mathrm{a} 2}=6.07\right)$. The maleic acid is more highly dissociated and as such is more hydrophilic and less likely to be incorporated into the micelle.

When a micelle is formed, there are two competing tendencies; the hydrophobic interaction between the tails promotes aggregation and the electrostatic or steric repulsion between headgroups oppose it. Together, the balance of these tendencies controls the size and shape of the micelle. As the level of headgroup repulsion is reduced, the structures adopt less curved geometries; this is described as an increase in the critical packing parameter. ${ }^{23} \mathrm{CTAB}$ adsorbs to silica in the form of flattened admicelles. ${ }^{24}$ When considering surface coadsorption, an increase in surface excess suggests that a structure more like a complete bilayer is formed. That is, the solutes that increase the surface excess are likely causing a decrease in the curvature of the surface of the admicelles by decreasing the effective headgroup area. Note that the effective headgroup area 
is always larger than the physical size of the headgroup for ionic surfactants because of like-charge repulsion. The results obtained in Figure 1 can be rationalized by changes in the effective headgroup area. When the solute is the benzene derivative, the headgroups of $\mathrm{CTAB}$ are pulled together by interactions with the $\pi$-electrons of benzene, leading to a decrease in the effective headgroup area and an increase in the value of the packing parameter. Indeed, NMR studies have already established that the aromatic ring of the salicylate molecule is preferably located between the tetramethylammonium headgroups of the surfactants, ${ }^{25}$ and cation $-\pi$ bonding has been demonstrated between tetramethylammonium headgroups and the aromatic groups of salicylate, benzoate, and phthalate. $^{26}$

The standard OR technique used here cannot distinguish between species that are adsorbing in a multicomponent system, though we note that Bain has developed a spectroscopic technique that can identify different chemical species using IR adsorption. ${ }^{15}$ Nonetheless, we would like to be able to determine the surface excess of $\mathrm{CTAB}$ and the solute when they are present together in solution. We are able to do this semiquantitatively by examining the adsorption isotherms obtained with different ratios of CTAB to solute in solution. Figure 2a shows the apparent adsorption isotherm for CTAB in the presence of $\mathrm{NaSal}$ for four different CTAB: $\mathrm{NaSal}$ ratios. We are interested in the high-concentration region of the isotherm where there are micelles present in solution. In the absence of any solute, a maximum in surface excess is reached and remains constant with an increasing surfactant concentration. However, in the presence of solute, we find that the apparent surface excess can increase, remain constant, or decrease above the $\mathrm{cmc}$ depending on the ratio of the surfactant to solute. For 5:1 and 1:1 CTAB:NaSal ratios, an apparent maximal surface excess of $\sim 3.2 \mathrm{mg} / \mathrm{m}^{2}$ is reached when the bulk concentration of CTAB is $0.25 \mathrm{mM}$ and then the surface excess declines with an increasing concentration. When a 1:5 CTAB:NaSal ratio is employed, the opposite trend is observed. That is, the apparent surface excess continues to increase with an increasing bulk concentration of CTAB. When a $1: 2$ CTAB:NaSal ratio is employed, the surface excess remains comparatively constant with an increasing surfactant concentration. Similar observations were made with other solutes previously ${ }^{12}$ and have been reported for CTAB:phenol ratios. ${ }^{22}$ The bottom panel of Figure 2 shows the adsorption isotherms for $\mathrm{CTAB}$ with phthalic acid as the solute at different CTAB:phthalic acid ratios. At a 5:1 CTAB:phthalic acid ratio, the apparent surface excess decreases from a maximum of $2.75 \mathrm{mg} / \mathrm{m}^{2}$ with an increasing concentration of CTAB; at a 1:1 CTAB:phthalic acid ratio, it remains constant, and a 1:5 ratio leads to a slight increase in surface excess.

The explanation for these isotherms lies in recognizing that above the $\mathrm{cmc}$ the admicelles are in equilibrium with micelles in solution and the solute partitions between them. ${ }^{1,12,22}$ Just as the admicelles consist of a mixture of $\mathrm{CTAB}$ and solute molecules, the micelles in solution will consist of $\mathrm{CTAB}$ and solute molecules. If the CTAB:solute ratio in solution is the same as the CTAB:solute ratio in the micelle, then the ratio of material in solution will not change with concentration. In cases where the ratio of solute to CTAB is higher in the micelle than in solution, then the solution will be relatively depleted of solute as the number of micelles increases. This will cause the solute to leave the admicelles, and a decrease in surface excess is seen. Conversely, in cases where the solute: $\mathrm{CTAB}$ ratio is lower
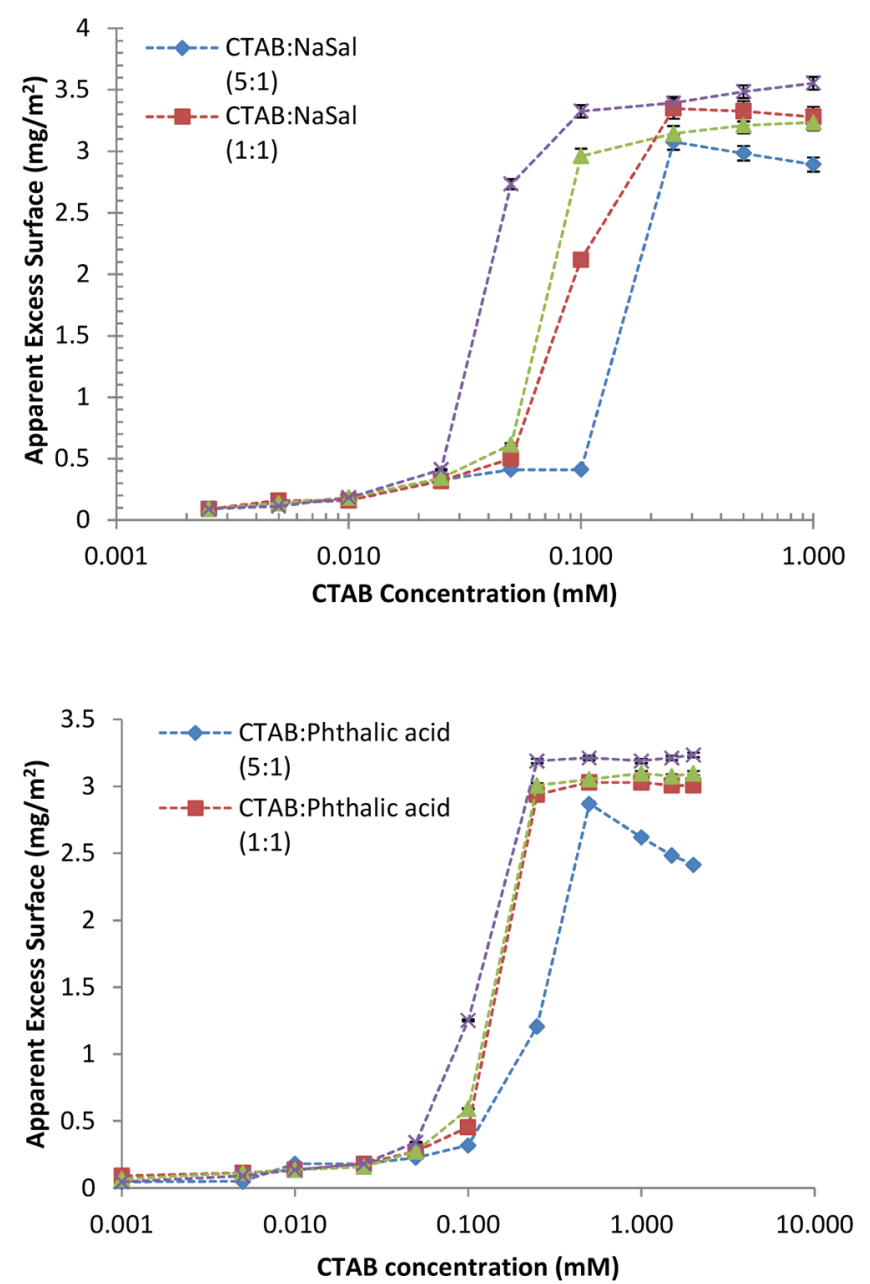

Figure 2. Apparent adsorption isotherm for CTAB in the presence of NaSal (top) and phthalic acid (bottom), presented on a linear-log scale. The apparent adsorption was calculated using the sensitivity parameter for $\mathrm{CTAB}\left(A_{\mathrm{S}}=-0.02195\right)$. The error bars were determined from repeated measurements, and some error bars are obscured by the symbols.

in the micelle than in the solution, the solution will be relatively enhanced in the solute. In this case, more solute will be incorporated into the admicelles and the surface excess will increase. In the case where there is no change in surface excess with an increasing concentration at a constant CTAB:solute ratio, the CTAB:solute ratio in the micelle is the same as that in solution. If we make the assumption that the CTAB:solute ratio in the admicelles is the same as in the micelles, then this process can be used to determine the CTAB:solute ratio in the admicelles. This is a reasonable approximation for silica surfaces that only weakly perturb the aggregate structure. We have measured the adsorption isotherms of CTAB with a range of solutes at different $\mathrm{CTAB}$ :solute ratios and determined the integer ratios that give rise to isotherms in which the surface excess remains approximately constant above the $\mathrm{cmc}$. As we now have an approximate measure of the CTAB:solute ratio in the admicelles, we can calculate the correct sensitivity parameter to use to convert the raw data to surface excess by appropriate weighting of the $\mathrm{d} n / \mathrm{d} c$ values of the two components (which are listed in Table S1 of the Supporting Information). It has previously been shown that the solute:CTAB ratio in the adsorbed film does not change with 
CTAB concentration. ${ }^{1}$ In Figure 3, we plot the combined surface excess of $\mathrm{CTAB}$ and solute for a range of solutes.

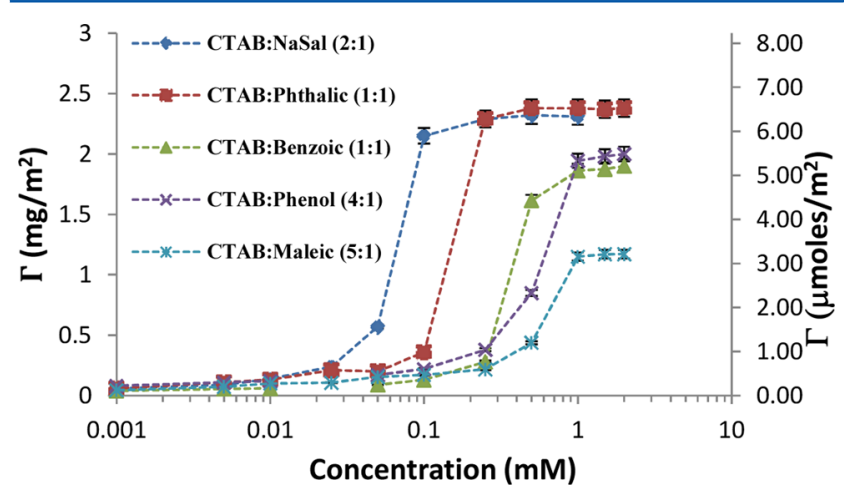

Figure 3. Adsorption isotherm for $\mathrm{CTAB}$ with five different solutes, including NaSal, phthalic acid, benzoic acid, phenol, and maleic acid, presented on a linear-log scale. The surface excess, $\Gamma$, was calculated from the sensitivity parameters of $C T A B$ and the solute, weighted by the appropriate ratio depending on the proportion of $C T A B$ to solute in the adsorbed layer (see the legend). The error bars were determined from repeated measurements. Note that some of the error bars cannot be discerned, as their size is comparable to the size of the symbols.

The next step in this process allows us to separate the contribution of CTAB from the solute to the overall surface excess. This is presented in panels $a$ and $b$ of Figure 4 for $\mathrm{CTAB}$ and solute, respectively. We find that solutes $\mathrm{NaSal}$ and phthalic acid at low concentrations enhance the adsorption of the CTAB onto the surface. We attribute this to a shift in the $\mathrm{cmc}$ to lower concentrations. This shifts the isotherms and the mcc to lower concentrations. This effect is very well-known for salicylate. $^{2-4}$ The other solutes have little to no effect on the mcc. It is important to note that at high $\mathrm{CTAB}$ concentrations the amount of $\mathrm{CTAB}$ on the surface is reduced in every case. Therefore, coadsorption generally leads to an overall increase in total surface excess but a decrease in the amount of CTAB adsorbed to the surface. This in part can be attributed to competition for space in the adsorbed film. We observe that there is a trend showing an inverse relationship between the amount of solute and the amount of CTAB in the films, with the exception of phthalic and benzoic acids whose order is reversed in this trend.

Our results for the $\mathrm{CTAB} / \mathrm{phenol}$ system are similar to those obtained previously, ${ }^{22}$ though these experiments are not directly comparable as we kept the species at a constant ratio whereas in the previous work phenol was held at a constant concentration.

NMR Experiments. The results presented above demonstrate that subtle changes in molecular architecture have a strong influence on the overall adsorption process, affecting both the amount of solute adsorbed and the amount of surfactant. Previous studies have established that small solutes with both hydrophilic and hydrophobic groups are primarily located in the interfacial region where they interact with both headgroups and the hydrocarbon core of micelles. We expect the same interaction with admicelles. It is clear that hydrophobic interactions, charge-charge interactions, and cation $-\pi$ bonding may all play a role. To improve our understanding of the specific chemical interactions at play, we have exploited the spatial and dynamic properties of NMR ROESY experiments. 1D ROESY experiments are commonly (a)

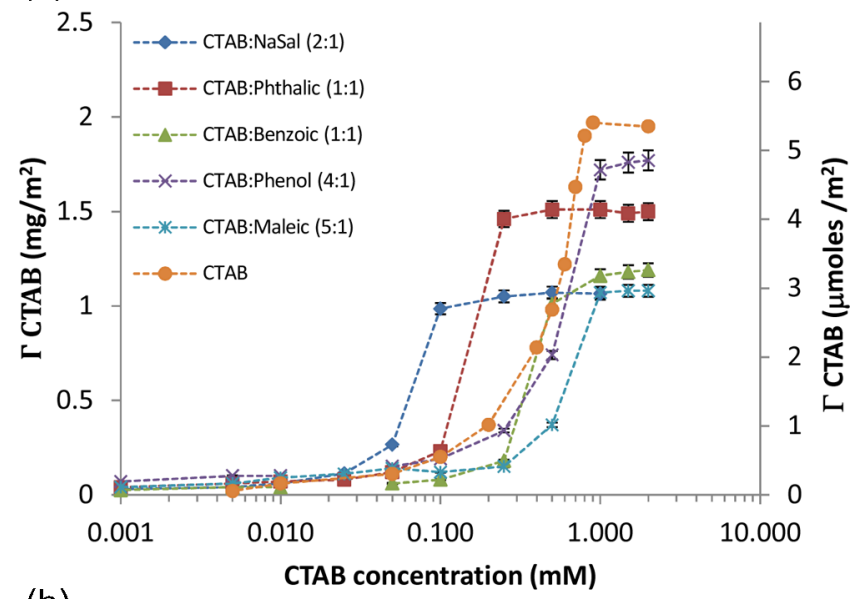

(b)

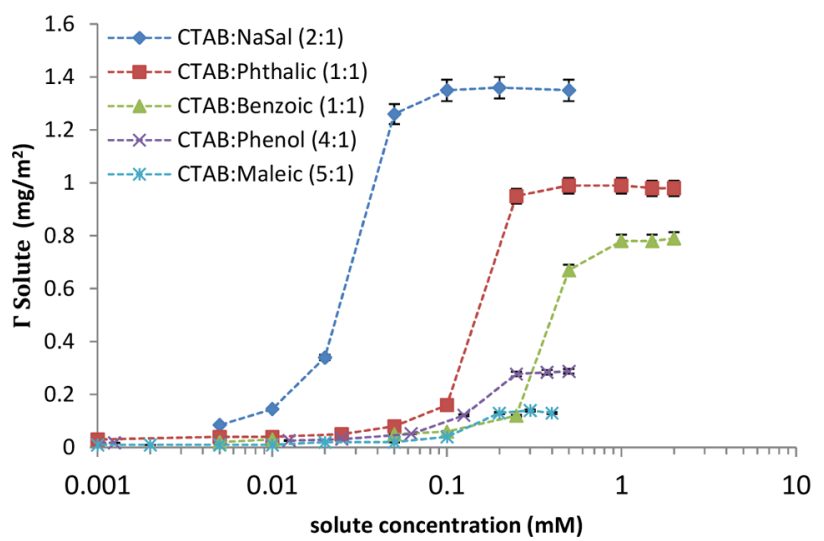

Figure 4. Surface excess of CTAB (a) and solutes (b) in CTAB/solute mixtures, presented on a linear-log scale. The apparent adsorption was calculated from the sensitivity parameter in Table S2 of the Supporting Information. The error bars were determined from repeat measurements. Some error bars are obscured by the symbols.

used with small to medium sized molecules to verify short through-space distance contacts $(<0.5 \mathrm{~nm})$ between protons especially if the effective correlation time is in the regime where the nuclear Overhauser effect (NOE) is near zero. In preliminary 1D NOESY experiments, we observed that intermolecular solute-substrate NOE peaks were weak or nonexistent, whereas in $1 \mathrm{D}$ ROESY experiments on the same sample, the ROE peaks were detectable. The size of the CTAB micelles would suggest that the rotational correlation time is in the regime where negative NOEs are expected, but internal motions of the solute within the micelle can result in a much shorter local correlation time, which affects the NOE and ROE intensities. Averaging of the intermolecular distances due to solute rotation, wobbling, and translation also reduces the observed NOE and ROE intensities. Because of the superior sensitivity of the 1D ROESY experiments, we recorded 1D ROESY spectra at an identical mixing time for each CTAB/ solute mixture. Without a detailed model for the motion of the solute within the micelle, it is possible to interpret the ROE intensity only in qualitative terms. The detection of ${ }^{1} \mathrm{H}-{ }^{1} \mathrm{H}$ intermolecular ROEs indicates that the individual solute and surfactant protons are close in space at least part of the time. The ROE, in this case, is a measure of the population-weighted average of the inverse sixth power of the interproton distances that are sampled. This average greatly favors the shortest 
distances in the ensemble because of the substantial ROE intensity at short distances. The ROE intensities also provide insight into the dynamic behavior within the micelle.

Representative 1D ROESY spectra of isophthalic acid, catechol, and NaSal dissolved in CTAB micelles will be discussed in some detail. The $\mathrm{CTAB}$ resonances were assigned by sequentially tracing the through-bond connectivities from the $\mathrm{E}$ resonance through to the $\mathrm{A} \mathrm{CH}_{3}$ resonance as shown in the 2D TOCSY spectrum located above the 1D ROESY spectra in Figure 5. The three 1D ROESY spectra of the CTAB/

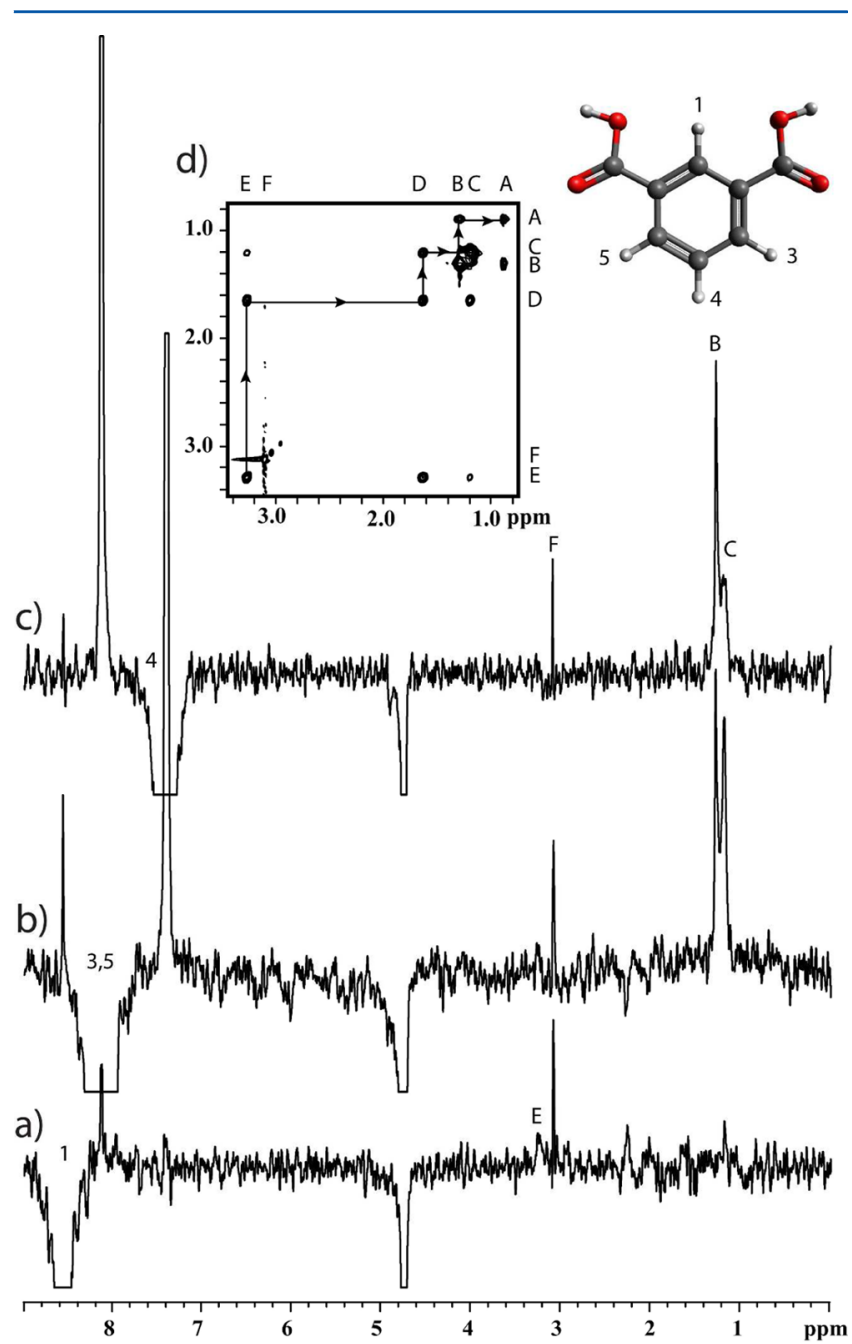

Figure 5. (a-c) 1D T-ROESY and (D) 2D zTOCSY spectra of CTAB in the presence of isophthalic acid. Each spectrum was recorded with the parameters given in the Experimental Section. The multiplets arising from (A) $\mathrm{H} 1,(B) \mathrm{H} 3$ and $\mathrm{H} 5$, and (C) $\mathrm{H} 4$ were selectively inverted with a $\mathrm{Q} 3$ pulse.

isophthalic acid mixture each demonstrate a distinctive ROE pattern. Selective excitation of $\mathrm{H} 1$ of isophthalic acid followed by a $200 \mathrm{~ms}$ ROESY mixing period generates ROEs at the $\mathrm{CTAB} E$ and $\mathrm{F}$ resonances (Figure 5a), indicating that $\mathrm{H} 1$ spends a substantial amount of time near the headgroup. A peak at the $\mathrm{F}$ resonance appears in most 1D ROESY spectra. We do not believe the signal at the $\mathrm{F}$ resonance is a reliable indication of the spatial proximity of the solute and CTAB molecules because the intensity pattern is not consistent with other ROE peaks. The fact that the F resonance sometimes has the phase that is the opposite of the phase of the other ROE peaks indicates there are either exchange or indirect ROE contributions to this peak. Because nine protons contribute to the peak intensity, peak $\mathrm{F}$ inherently has a large intensity relative to those of other peaks. Selective excitation of the unresolved $\mathrm{H} 3$ and $\mathrm{H} 5$ resonances followed by ROESY mixing generates ROEs at the CTAB $\mathrm{B}$ and $\mathrm{C}$ resonances (Figure $5 \mathrm{~b}$ ), as does selective excitation at $\mathrm{H} 4$, although the $\mathrm{ROE}$ at the $\mathrm{CTAB} \mathrm{B}$ resonance is more prominent in the latter spectrum (Figure $5 c$ ). The pattern of ROEs in Figure $5 a-c$ is consistent with an isophthalic acid orientation that places the carboxylates near the headgroups of the surfactant and $\mathrm{H} 4$ nearest the center of the micelle. However, the uniformly increased intensity of the intermolecular isophthalic-CTAB ROEs compared to those of all the other solutes (Table 1) suggests that, compared

Table 1. Intermolecular Rotating Frame Overhauser Enhancements (ROEs) between the CTAB Protons and the Solute Protons ${ }^{b}$

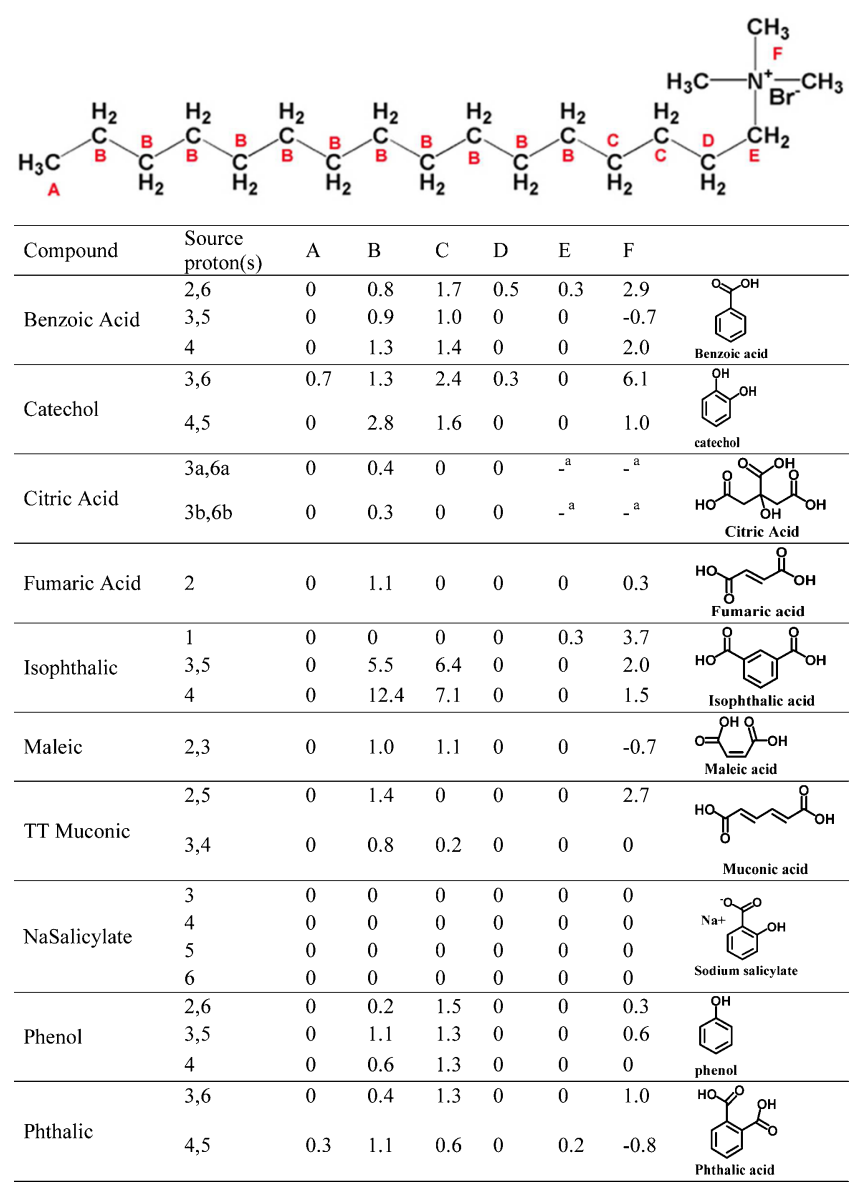

${ }^{a}$ Could not be measured because of overlap with the source peak. ${ }^{b}$ The ROEs are given as arbitrary intensities normalized relative to the integrated intensity of the source peak, which is set to a value of -1000 in each case. All ROEs were recorded for a T-ROESY mixing time of $200 \mathrm{~ms}$.

to that of the other solutes, the isophthalic internal motion is slowed by an arrangement of the acid groups that promotes an interaction between two surfactant molecules. However, the similarity in the surface excess obtained with isophthalic and phthalic acid as solutes suggests that the particular arrangement of the carboxylate groups is unimportant for coadsorption.

If we take phthalic acid and exchange the carboxylate groups for hydroxyl groups, we obtain catechol. Therefore, by 
comparing these molecules, we can gain a sense of the importance of the charge on the carboxylate groups to the interaction with the surfactant headgroups. Indeed, we find that the level of adsorption is lower for catechol, and one might expect that is due merely to the lack of charge on the catechol molecule. Indeed, the CTAB/catechol 1D ROESY spectra (Figure 6) are consistent with an orientation that places the

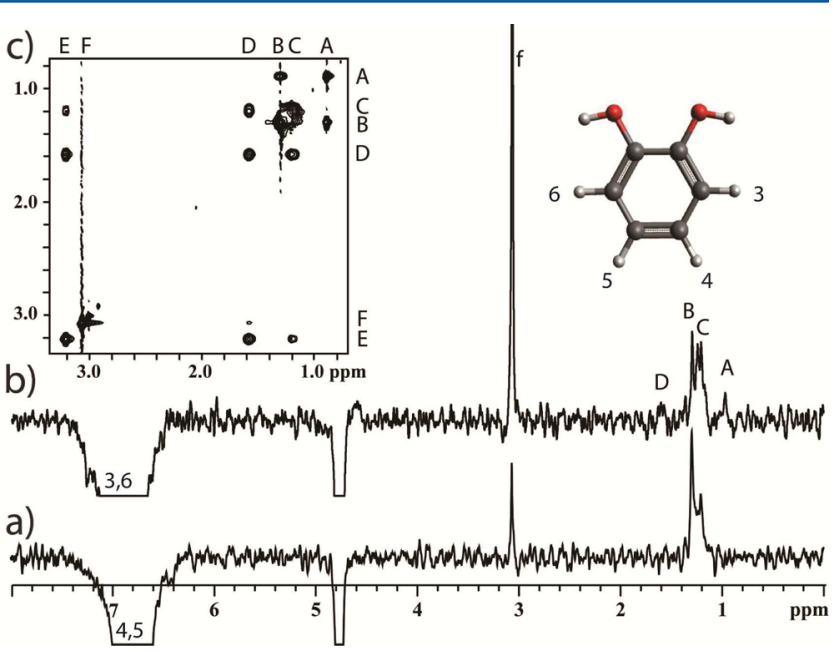

Figure 6. (a and b) 1D ROESY and (c) 2D zTOCSY spectra of CTAB in the presence of catechol. Each spectrum was recorded with the parameters given in the Experimental Section. The multiplets arising from (a) $\mathrm{H} 4$ and $\mathrm{H} 5$ and (b) $\mathrm{H} 3$ and $\mathrm{H} 6$ were selectively inverted with a Q3 pulse.

phenolic group near the headgroup and the $\mathrm{H} 4$ and $\mathrm{H} 5$ protons nearer the center of the micelle. However, the ROE pattern upon excitation of the $\mathrm{H} 3$ and $\mathrm{H} 6$ resonances is not consistent with a linear conformation for all $\mathrm{CTAB}$ methylene chains (Figure 6b). In addition to an ROE contact between $\mathrm{H} 3$ and $\mathrm{H} 6$ of the catechol and the $\mathrm{B}$ and $\mathrm{C}$ protons of $\mathrm{CTAB}$, there are catechol 3,6 ROE contacts with the $\mathrm{D}$ and A protons, but ROE contacts between the catechol 4 and 5 protons and the CTAB $\mathrm{D}$ and A protons are weak or missing. This ROE pattern is only possible if the catechol molecule is free to rotate about an axis that bisects the phenolic groups and $\mathrm{C} 4$ and $\mathrm{C} 5$ and a part of the population of CTAB chains that bends back and surrounds the aromatic ring as shown in Figure 7. Given this interaction with the hydrocarbon chain and the proximity of the catechol molecule to the headgroups, one might expect catechol to have

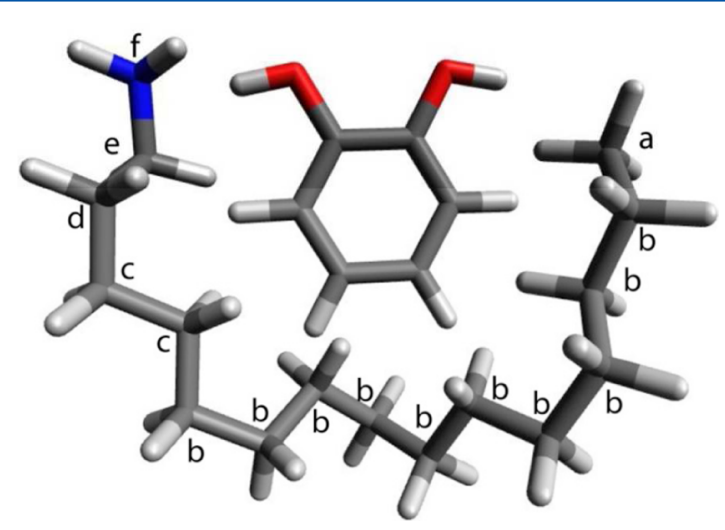

Figure 7. Diagram of catechol and a CTAB molecule that satisfies the observed ROEs in parts a and b of Figure 6. a dramatic effect on interfacial curvature and therefore the structure of $\mathrm{CTAB} /$ catechol micelles and admicelles. No such dramatic influence is observed. This can be rationalized by considering the fluidity of the hydrocarbon core of micelles and admicelles. While some alkane chains will be preferentially associated with the catechol molecule, others will be extended in an all-trans arrangement. An increased number of trans chains must compensate for the influence of catechol such that there is not a large alteration to the overall curvature of the interface. The conclusion is that the rearrangements of the chains within the hydrophobic core do not result in changes to the admicelle or micellar structure; this requires a change in the overall volume. Previously, changes in the chemical shift of the $\mathrm{CTAB}$ along the whole chain have been explained in terms of transient migration of the solute as far as the center of the micelle, but these chemical shift changes are also consistent with a preferred solute location near the headgroup and transient movement of the terminus of the $\mathrm{CTAB}$ methylene chain toward the headgroup.

The solute salicylate is known to have dramatic effects on the rheology and structure of $\mathrm{CTAB}$ aggregates, and we also find that it has the strongest influence on adsorption. Significantly, the NMR spectra of the CTAB/NaSal mixture are atypical of the spectra of all the other mixtures. The $\mathrm{CTAB}$ resonances are broad, which is clear from inspection of the 2D TOCSY spectrum (Figure $8 \mathrm{~d}$ ). The extreme broadening of the CTAB resonances is explained in terms of the much slower tumbling

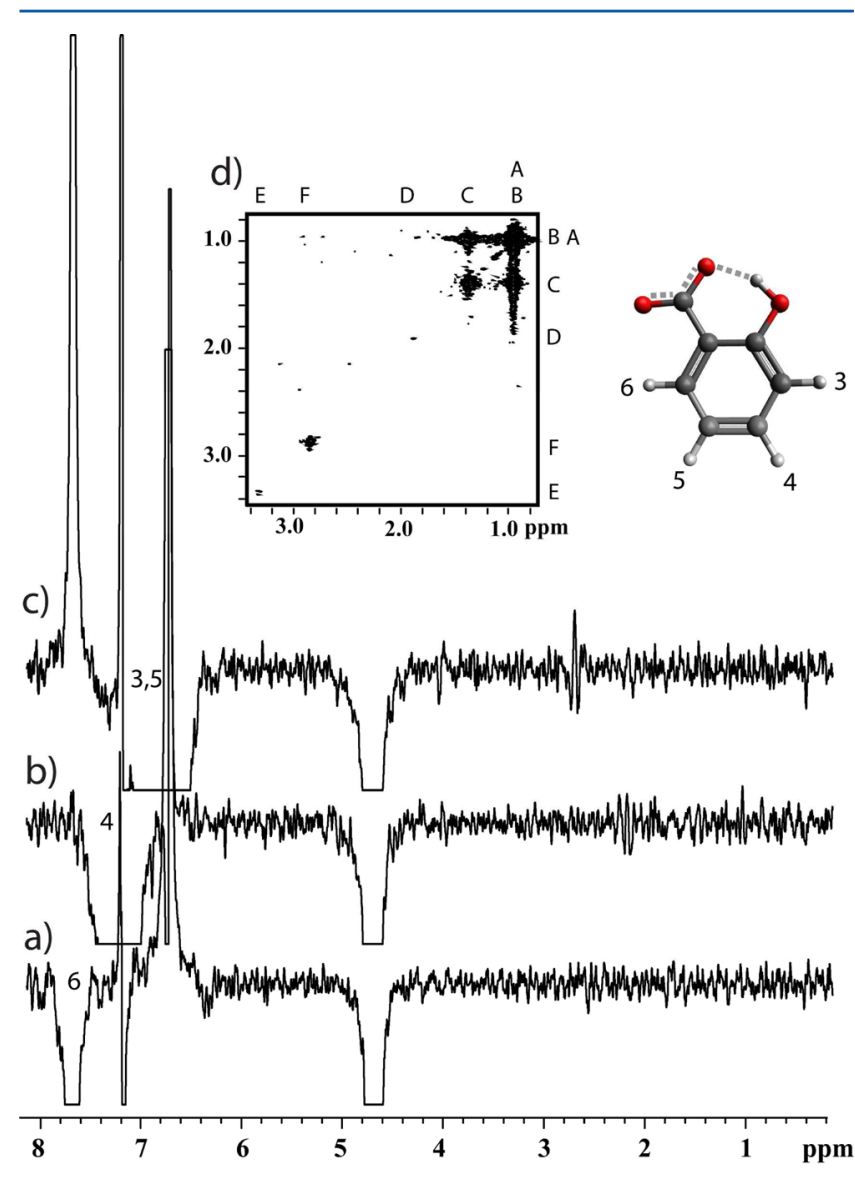

Figure 8. $(a-c) 1 D$ ROESY and (d) 2D zTOCSY spectra of CTAB in the presence of NaSal. The spectra were recorded with the parameters given in Experimental Section. The multiplets arising from (a) H6, (b) $\mathrm{H} 4$, and (c) H3 and H5 were selectively inverted with a Q3 pulse. 
of large wormlike micelles compared to that of the smaller spherical CTAB micelles. The NaSal peaks are substantially narrower than the $\mathrm{CTAB}$ peaks but broader than the peaks of solute molecules dissolved in the CTAB micelles of corresponding mixtures. The differential broadening has been observed previously ${ }^{27}$ and can be explained in terms of an additional reorientation of the $\mathrm{NaSal}$ molecule that is independent of the slow tumbling of the wormlike CTAB micelle. Anet ${ }^{27}$ described the independent motion in terms of a rapid rotation about the $\mathrm{C} 1-\mathrm{C} 4$ axis of $\mathrm{NaSal}$, which explained the unusual multiplet structures of the $\mathrm{NaSal}{ }^{1} \mathrm{H}$ spectrum and the differential relaxation mechanisms that he observed. We observe multiplet structures similar to those of Anet ${ }^{27}$ and intramolecular ROEs between the NaSal protons. However, we do not observe any ROEs between $\mathrm{NaSal}$ protons and CTAB protons (Figure $8 \mathrm{a}-\mathrm{c}$ ). Consequently, it is not possible to locate the position of $\mathrm{NaSal}$ in the CTAB micelles by this technique. It is unclear whether the absence of ROEs is the result of poor signal strength because of the broad CTAB resonances, an ill-defined NaSal location within the micelles, self-association of $\mathrm{NaSal}$ within the micelles, or a combination of all of these. On the basis of the current evidence, we feel the most likely explanation is that the salicylate anions are dimerizing. As the aromatic groups can interact with the headgroup cations, such dimerization could effectively link two headgroups in an arrangement whereby the aromatic groups of the salicylate ions are closely associated with headgroups while the salicylate ions are linked through hydrogen bonding into pairs. This model is consistent with the large adsorption of salicylate and would explain the dramatic decrease in the effective headgroup area that is required to produce an increase in the critical packing parameter ${ }^{23}$ required for the transition to wormlike micelles at low surfactant concentrations. A schematic of this arrangement is depicted in Figure 9. We are currently testing this proposal by looking for further evidence of dimerization of salicylate molecules by other means.

Table 1 summarizes the intermolecular ROEs of all the $\mathrm{CTAB} /$ solute systems that we have studied. Although we believe there is a certain degree of mobility of the solutes within the micelles, the data indicate that the aromatic molecules have a favored orientation that places the hydrophilic part of the molecule close to the headgroup and the hydrophobic part nearer the center of the micelle, consistent with previous NMR studies. ${ }^{12,13}$ We have observed ROE contacts with the terminal methyl group in the 1D ROESY spectra of catechol and phthalic acid, which suggests that at any point in time a part of the population of $\mathrm{CTAB}$ molecules bends back to surround the aromatic ring. Although we do not observe ROE contacts between the methyl group and protons of other aromatic solutes, this does not preclude the possibility of the CTAB chain from bending back in these other systems. The strongest ROE contacts between the nonexchangeable protons in the nonaromatic solutes are with the CTAB B protons. This implies that the small nonaromatics reside deeper within the micelle than the aromatic solutes. Aromatic groups can interact with the headgroups through cation $-\pi$ bonding. The lack of this interaction for the nonaromatics will see the hydrophobic interactions become more important and lead to a deeper positioning within the micelles and admicelles.

\section{CONCLUSIONS}

Coadsorption of small solute molecules with the cationic surfactant $\mathrm{CTAB}$ is complex. When the molecules have both

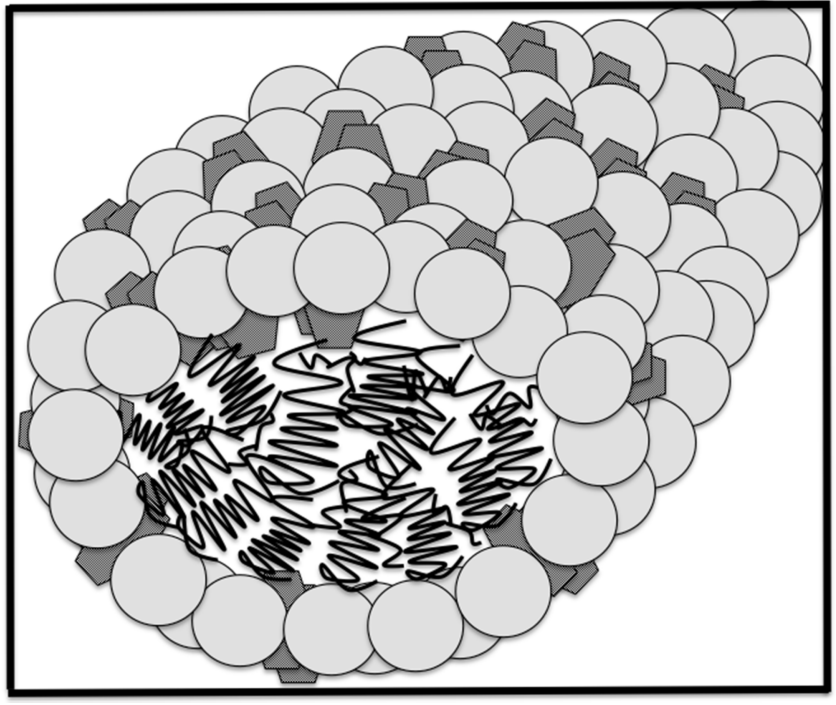

Figure 9. Schematic showing the arrangement of salicylate molecules in wormlike micelles formed from mixtures of $\mathrm{CTAB}$ and salicylate. The adsorption studies suggest that two salicylate ions are present for each $\mathrm{CTAB}$ ion. The NMR evidence suggests that the salicylate forms dimers. We propose that these dimers interact with two cationic surfactant headgroups through cation $-\pi$ bonding. This brings the headgroups closer together and lowers the effective headgroup area, resulting in an increase in the critical packing parameter and the elongation of the micelle structure.

hydrophilic and hydrophobic regions, they tend to reside in the interfacial region to satisfy hydrophobic and hydrophilic interactions. Additionally, aromatic molecules strongly interact with the surfactant headgroups through cation $-\pi$ bonding, which favors adsorption and reduces the curvature of the aggregates by bringing surfactant headgroups closer together. Additionally, the specific orientation of chemical groups in similar molecules leads to large changes in macroscopic properties. We propose a new model for the interaction of the salicylate anion with $\mathrm{CTAB}$, in which dimers of salicylate interact with neighboring $\mathrm{CTAB}$ molecules through cation $-\pi$ bonding and in doing so act as a chelating agent. This lowers the effective headgroup area, decreasing the interfacial curvature and promoting the formation of wormlike micelles, which are viscoelastic. Differences in the interactions of molecules with the hydrocarbon chains of the surfactant have little or no effect on the surfactant aggregate structure. This is consistent with the model of a micelle core effectively being an oil-like phase.

\section{ASSOCIATED CONTENT}

\section{S Supporting Information}

$\mathrm{d} n / \mathrm{d} c$ values and sensitivity parameters of CTAB and all solute molecules employed in OR experiments, sensitivity parameters used for $\mathrm{CTAB}$ with solutes, and measured surface excesses of solute molecules adsorbed to silica in the absence of CTAB. This material is available free of charge via the Internet at http://pubs.acs.org.

\section{AUTHOR INFORMATION}

\section{Corresponding Author}

*E-mail: vince.craig@anu.edu.au.

\section{Notes}

The authors declare no competing financial interest. 


\section{ACKNOWLEDGMENTS}

T.W. is grateful for the award of a 2013 Endeavour Research Scholarship from the Department of Industry, Innovation, Science, Research and Tertiary Education of the Australian Government. V.S.J.C. gratefully acknowledges the support of the Australian Research Council through a Future Fellowship (FT09919933).

\section{REFERENCES}

(1) Aloulou, F.; Boufi, S.; Belgacem, N.; Gandini, A. Adsorption of cationic surfactants and subsequent adsolubilization of organic compounds onto cellulose fibers. Colloid Polym. Sci. 2004, 283, 344-350.

(2) Gravsholt, S. Viscoelasticity in highly dilute aqueous-solutions of pure cationic detergents. J. Colloid Interface Sci. 1976, 57, 575-577.

(3) Shikata, T.; Hirata, H.; Kotaka, T. Micelle formation of detergent molecules in aqueous-media: Viscoelastic properties of aqueous cetyltrimethylammonium bromide solutions. Langmuir 1987, 3, $1081-1086$.

(4) Bijma, K.; Rank, E.; Engberts, J. Effect of counterion structure on micellar growth of alkylpyridinium surfactants in aqueous solution. $J$. Colloid Interface Sci. 1998, 205, 245-256.

(5) Rao, U. R. K.; Manohar, C.; Valaulikar, B. S.; Iyer, R. M. Micellar chain model for the origin of the visoelasticity in dilute surfactant solutions. J. Phys. Chem. 1987, 91, 3286-3291.

(6) Shikata, T.; Hirata, H.; Kotaka, T. Micelle formation of detergent molecules in aqueous-media. 3. viscoelastic properties of aqueous cetyltrimethylammonium bromide salicylic-acid solutions. Langmuir 1989, 5, 398-405.

(7) Li, L.; Du, X.; Kou, J.; Liu, X.; Lu, Y.; Yang, Z. Study on the interaction between dihydroxybenzenes and DTAB by ultramicroelectrode voltammetry. Colloids Surf., A 2008, 322, 219-224.

(8) Srinivasan, V.; Blankschtein, D. Effect of counterion binding on micellar solution behavior: 2 . Prediction of micellar solution properties of ionic surfactant-electrolyte systems. Langmuir 2003, 19, 99469961.

(9) Shapley, W. A.; Bacskay, G. B.; Warr, G. G. Ab initio quantum chemical studies of the $\mathrm{pK}_{\mathrm{a}}$ 's of hydroxybenzoic acids in aqueous solution with special reference to the hydrophobicity of hydroxybenzoates and their binding to surfactants. J. Phys. Chem. B 1998, 102, 1938-1944.

(10) Howard, S. C.; Craig, V. S. J. Very slow surfactant adsorption at the solid-liquid interface is due to long lived surface aggregates. Soft Matter 2009, 5, 3061-3069.

(11) Wangchareansak, T.; Craig, V. S. J.; Notley, S. M. Adsorption Isotherms and Structure of Cationic Surfactants Adsorbed on Mineral Oxide Surfaces Prepared by Atomic Layer Deposition. Langmuir 2013, $29,14748-14755$.

(12) Howard, S. C.; Craig, V. S. J. Adsorption of the cationic surfactant $\mathrm{CTAB}$ to silica in the presence of sodium salicylate: Surface excess and kinetics. Langmuir 2009, 25, 13015-13024.

(13) Wanless, E. J.; Ducker, W. A. Organization of sodium dodecyl sulfate at the graphite-solution interface. J. Phys. Chem. 1996, 100, 3207-3214

(14) Ducker, W. A.; Wanless, E. J. Adsorption of hexadecyltrimethylammonium bromide to mica: Nanometer-scale study of bindingsite competition effects. Langmuir 1999, 15, 160-168.

(15) Tyrode, E.; Rutland, M. W.; Bain, C. D. Adsorption of CTAB on Hydrophilic Silica Studied by Linear and Nonlinear Optical Spectroscopy. J. Am. Chem. Soc. 2008, 130, 17434-17445.

(16) Dijt, J. C.; Stuart, M. A. C.; Fleer, G. J. Reflectometry as a tool for adsorption studies. Adv. Colloid Interface Sci. 1994, 50, 79-101.

(17) Howard, S. C.; Atkin, R.; Craig, V. S. J. Effect of electrolyte species on the adsorption of a cationic surfactant to silica: The common intersection point. Colloids Surf., A 2009, 347, 109-113.

(18) Howard, S. C.; Craig, V. S. J. Very slow surfactant adsorption at the solid-liquid interface is due to long lived surface aggregates. Soft Matter 2009, 5, 3061.
(19) Azzam, R. M. A.; Bashara, N. M. Ellipsometry and polarized light; Elsevier Science Publishing Co.: Amsterdam, 1987; p xvii.

(20) Hwang, H.-L.; Shaka, A. J. Cross relaxation without TOCSY. J. Am. Chem. Soc. 1992, 114, 3157-3159.

(21) Atkin, R.; Craig, V. S. J.; Biggs, S. Adsorption kinetics and structural arrangements of cationic surfactants on silica surfaces. Langmuir 2000, 16, 9374-9380.

(22) Schieder, D.; Dobias, B.; Klumpp, E.; Schwuger, M. J. Adsorption and solubilization of phenols in the hexadecyltrimethylammonium chloride adsorbed layer on quartz and corundum. Colloids Surf., A 1994, 88, 103-111.

(23) Israelachvili, J. N.; Mitchell, D. J.; Ninham, B. W. Theory of selfassembly of hydrocarbon amphiphiles into micelles and bilayers. J. Chem. Soc., Faraday Trans. 2 1976, 72, 1525-1568.

(24) Fleming, B. D.; Biggs, S.; Wanless, E. J. Slow Organization of Cationic Surfactant Adsorbed to Silica from Solutions Far below the CMC. J. Phys. Chem. B 2001, 105, 9537-9540.

(25) Shikata, T.; Hirata, H.; Kotaka, T. Micelle formation of detergent molecules in aqueous-media. 2. Role of free salicylate ions on viscoelastic properties of aqueous cetyltrimethylammonium bromide sodium-salicylate solutions. Langmuir 1988, 4, 354-359.

(26) Wu, H. W.; Kawaguchi, S.; Ito, K. H-1 NMR studies on intermolecular association of amphiphilic cationic polyelectrolyte micelles induced by hydrophobic counteranions in water. Colloid Polym. Sci. 2005, 283, 636-645.

(27) Anet, F. A. L. Novel spin-spin splitting and relaxation effects in the proton NMR spectra of sodium salicylate in viscoelastic micelles. J. Am. Chem. Soc. 1986, 108, 7102-7103. 